EPJ Web of Conferences 73, 01011 (2014)

DOI: 10.1051/epjconf/20147301011

(C) Owned by the authors, published by EDP Sciences, 2014

\title{
Time-reversal violation in the nucleon and light nuclei
}

\author{
U. van Kolck ${ }^{1,2, a}$ \\ ${ }^{1}$ Institut de Physique Nucléaire, CNRS/IN2P3, Université Paris Sud, 91406 Orsay, France \\ ${ }^{2}$ Department of Physics, University of Arizona, Tucson, AZ 85721, USA
}

\begin{abstract}
I review the effective-field theoretical framework for disentangling contributions to the electric dipole moments of the nucleon and light nuclei from physics in and beyond the Standard Model.
\end{abstract}

\section{Introduction}

It feels like a sin to speak about time-reversal $(T)$ violation (TV) in the Eternal City. Still I am grateful for the opportunity to convey a message regarding a proposed experimental program to measure electric dipole moments (EDMs) of the nucleon and light nuclei. Searches for the neutron EDM have a long and venerable history that has resulted in a tight bound, $\left|d_{n}\right|<2.9 \cdot 10^{-13} e \mathrm{fm}$ [1], and in realistic promises of improvements by one or two orders of magnitude over the next few years. Even more exciting, there are proposals to probe the EDMs of light ions at storage rings, which might lead to sensitivity to the proton $\left(d_{p}\right)$, deuteron $\left(d_{d}\right)$ and helion $\left(d_{h}\right)$ EDMs at similar levels [2]. As argued below, a measurement of the triton $\operatorname{EDM}\left(d_{t}\right)$ would be useful as well.

Why do I find this so exciting? We already know that $T$ is not an exact symmetry because of the nonvanishing phase of the CKM matrix, which, however, does not seem enough to generate the observed matter-antimatter asymmetry of the universe. Thus, other interactions must exist in the Standard Model (SM) and beyond (BSM) that cause TV, such as the QCD vacuum angle $\bar{\theta}$. Since the phase of the CKM matrix gives contributions to neutron and light nuclear EDMs $\sim 10^{-19} e \mathrm{fm}$, any signal in the next generations of experiments will mean new physics. The question my collaborators and I have addressed is: Should a signal be found, what measurements would allow us to infer the underlying TV mechanism(s)?

We can answer this question using the framework of effective field theories (EFTs). The SM is likely an EFT at the electroweak scale $M_{W} \sim 100 \mathrm{GeV}$, where the well-known dimension-four operators are accompanied by operators of higher dimension involving the known degrees of freedom and symmetries, but suppressed by powers of a larger mass scale. In general we expect the lowest-dimension operators to be most important, and it is indeed very possible that neutrino masses, as well as sources of leptonic $\mathrm{TV}$, originate in the only allowed dimension-five operator. Among the many dimension-six operators, several are TV and suppressed by $M_{T}^{-2}$, where $M_{T}$ denotes the mass scale of the new physics.

\footnotetext{
ae-mail: vankolck@ipno.in2p3.fr
}

This is an Open Access article distributed under the terms of the Creative Commons Attribution License 4.0, which permits unrestricted use, distribution, and reproduction in any medium, provided the original work is properly cited. 
In the EFT below $M_{W}$, the TV sources involving quarks and gluons, on which I focus, appear as operators that can be interpreted as quark EDMs (qEDMs) and color-EDMs (qCEDMs), a gluon color-EDM (gCEDM), two chiral-invariant four-quark operators (CIFQ), and two left-right four-quark operators (LRFQ) that stem from a single two-Higgs/quark interaction. Ordinarily one would expect them to be negligible compared to the dimension-four $\bar{\theta}$ term. However, one can infer from the current neutron EDM bound (using the techniques described below) that $\bar{\theta} \lesssim 10^{-10}$. Thus, dimension-six operators might be competitive. Just a neutron EDM signal, while groundbreaking, would not in itself tell us much about the new TV source(s), but, as I will argue below, light nuclear EDMs would.

The crucial point is that the approximate chiral symmetry of QCD acts like a filter through which we can separate the effects of the $\bar{\theta}$ term and the dimension-six sources. At momenta below the characteristic QCD scale $M_{Q C D} \sim 1 \mathrm{GeV}$, TV can be incorporated [3-5] in chiral EFT [6], which generalizes chiral perturbation theory [7] to the multinucleon sector and serves as the basis for nuclear calculations consistent with the rest of the SM.

I will focus here on the sequence of EFTs and the naive dimensional analysis (NDA) that allow model-independent, albeit only semi-quantitative, statements. I emphasize the calculations of nucleon EDMs up to next-to-leading order (NLO) [8, 9] and light-nuclear EDMs to leading order (LO) [10-13]. A more complete review of this research program, including calculations of hadronic matrix elements, Schiff moments of heavier nuclei, atomic/molecular EDMs, specific models of BSM physics, and further references to the literature can be found in Ref. [14].

\section{Effective field theories}

An EFT accounts for the most general dynamics among the degrees of freedom relevant at the momentum scale of interest $Q$, constrained only by symmetries. The interaction strengths, or "lowenergy constants" (LECs), carry information about the details of the physics at the higher scale $M$, which is not considered explicitly in the theory. Observables are expanded in the ratio $Q / M$. I will look at TV as $M$ is decreased.

I assume that at the scale $M_{W}$ the degrees of freedom are just the known quarks, leptons, gauge bosons, and the recently detected Higgs boson, with interactions constrained by the $S U(3)_{C} \times$ $S U(2)_{L} \times U(1)_{Y}$ gauge group of the SM. The up and down-type right-handed quarks are denoted $u_{R}$ and $d_{R}$, while the left-handed ones can be assembled in a weak-isospin doublet $q_{L}^{I}, I=1,2$. Quarks couple - with a fine-structure constant $\alpha_{s}=g_{s}^{2} /(4 \pi)$ - via the Gell-Mann $S U(3)$ matrices $\lambda^{a}, a=$ $1, \ldots, 8$ (with structure constants $f^{a b c}$ ) to gluons of field strength $G_{\mu \nu}^{a}$. ( $\tilde{G}_{\mu \nu}^{a} \equiv \epsilon_{\mu \nu \alpha \beta} G^{a \alpha \beta} / 2$.) Quarks also couple - with a fine-structure constant $\alpha_{e m}=e^{2} /(4 \pi)$ - to photons of field strength $F_{\mu \nu}$. The Higgs field is assumed to form a weak-isospin doublet of scalar fields $\varphi^{I}, I=1,2$; I use the shorthand notation $\tilde{\varphi}^{I}=\epsilon^{I J} \varphi^{J *}$, where $\epsilon^{I J}$ is the antisymmetric tensor in two dimensions. I denote the gauge covariant derivative as $D_{\mu}$ and the Yukawa couplings to up and down quarks as $Y_{u}$ and $Y_{d}$. Focusing on the lightest quark family, from the complete list of dimension-six operators [15], we can identify those relevant for TV [5]:

$$
\begin{aligned}
\mathcal{L}_{E W, P T}= & -\bar{\theta} \frac{g_{s}^{2}}{32 \pi} G_{\mu \nu}^{a} \tilde{G}^{a \mu \nu}+w \frac{g_{s}}{3 M_{T}^{2}} f^{a b c} G_{\mu \nu}^{a} G_{\rho}^{b \nu} \tilde{G}^{c \mu \rho}-\frac{(4 \pi)^{2}}{M_{T}^{2}} \xi\left(\bar{u}_{R} \gamma^{\mu} d_{R} \tilde{\varphi}^{\dagger} D_{\mu} \varphi+\text { H.c. }\right) \\
& -\frac{g_{s}}{2 M_{T}^{2}}\left\{\bar{q}_{L} \sigma^{\mu \nu} \lambda^{a}\left[Y_{u}\left(\tilde{\delta}_{0}+\tilde{\delta}_{3}\right) \tilde{\varphi} u_{R}+Y_{d}\left(\tilde{\delta}_{0}-\tilde{\delta}_{3}\right) \varphi d_{R}\right]+\text { H.c. }\right\} G_{\mu \nu}^{a} \\
& -\frac{e}{2 M_{T}^{2}}\left\{\bar{q}_{L} \sigma^{\mu \nu}\left[Y_{u}\left(\delta_{0}+\delta_{3}\right) \tilde{\varphi} u_{R}+Y_{d}\left(\delta_{0}-\delta_{3}\right) \varphi d_{R}\right]+\text { H.c. }\right\} F_{\mu \nu} \\
& +\frac{(4 \pi)^{2}}{M_{T}^{2}} \epsilon_{J K}\left(i \sigma_{1} \bar{q}_{L}^{J} u_{R} \bar{q}_{L}^{K} d_{R}+i \sigma_{8} \bar{q}_{L}^{J} \lambda^{a} u_{R} \bar{q}_{L}^{K} \lambda^{a} d_{R}+\text { H.c. }\right)+\ldots,
\end{aligned}
$$


where $w, \xi, \tilde{\delta}_{0,3}, \delta_{0,3}$, and $\sigma_{1,8}$ are dimensionless LECs whose values depend on the BSM physics, and “..." include heavier quarks and leptons, and terms whose effects are smaller at low energies. It is remarkable that all of the interactions in Eq. (1) are also parity $(P)$ violating $(\mathrm{PV})$, so generically we expect that $P$-conserving (PC) TV observables should be further suppressed.

As we go below $M_{W}$, the Higgs acquires a vacuum expectation value, which gives the lightest up and down quarks masses $\bar{m}(1-\varepsilon)$ and $\bar{m}(1+\varepsilon)$, respectively. By a chiral rotation, the $\bar{\theta}$ term can be transformed [16] into a complex quark mass proportional to $\bar{\theta} \bar{m}\left(1-\varepsilon^{2}\right) / 2$ when $\bar{\theta}$ is small. The terms with LECs $\tilde{\delta}_{0,3}\left(\delta_{0,3}\right)$ generate isoscalar and isovector qCEDMs (qEDMs). The $w$ term can be thought of as the gCEDM. The only TV four-quark interactions (CIFQ) have the LECs $\sigma_{1,8}$, except that, once we integrate out the weak gauge bosons, $W$-boson exchange between quarks coming from the dimensionfour $T$-conserving (TC) left-handed coupling and from the term with LEC $\xi$ generates a LRFQ. The running of PVTV operators is discussed in Ref. [17].

At the scale $M_{Q C D} \sim 1 \mathrm{GeV}$, the relevant degrees of freedom in the strong-interacting sector are the lightest quarks, gluons, and the photon. The dominant PCTC dynamics has an approximate chiral $S U(2)_{L} \times S U(2)_{R} \sim S O(4)$ symmetry of independent rotations of left- and right-handed quarks. The symmetry is broken explicitly by the quark masses as the sum of two $S O(4)$ vectors. It can be shown [3] that the $\bar{\theta}$ term breaks chiral symmetry through one of these vectors, which means a connection with quark-mass isospin violation. Similarly [5], the qCEDMs and qEDMs break chiral symmetry as $S O(4)$ vectors, the LRFQ transforms as a sum of rank-two $S O(4)$ tensors, and gCEDM and CIFQ are $S O(4)$ singlets.

Below $M_{Q C D}$ the most relevant degrees of freedom are instead the isovector pions $\pi$ and the isodoublet of bi-spinor nucleons $N$, which have velocity $v^{\mu}$, spin $S^{\mu}$ and isospin $\tau_{i} / 2, i=1,2,3$. The nucleon is non-relativistic because it has mass $m_{N}=\mathcal{O}\left(M_{Q C D}\right)$. Pions are light because they are the pseudo-Goldstone bosons of the spontaneous breaking of chiral symmetry, characterized by the pion decay constant $F_{\pi} \simeq 185 \mathrm{MeV}$. Chiral symmetry plays an important role in determining the form and relative importance of the effective interactions. Among other effects, its explicit breaking leads to a pion mass $m_{\pi}=\mathcal{O}\left(M_{Q C D} \bar{m}\right)$ and a neutron-proton mass splitting $\delta m_{N}=\mathcal{O}(\varepsilon \bar{m})$. The resulting PVTV Lagrangian is [3-5]

$$
\begin{aligned}
\mathcal{L}_{\chi, P T}= & -2 \bar{N}\left(\bar{d}_{0}+\bar{d}_{1} \tau_{3}\right) S_{\mu} N v_{\nu} F^{\mu \nu}-\frac{1}{F_{\pi}} \bar{N}\left(\bar{g}_{0} \tau \cdot \pi+\bar{g}_{1} \pi_{3}\right) N \\
& +\bar{C}_{1} \bar{N} N \partial_{\mu}\left(\bar{N} S^{\mu} N\right)+\bar{C}_{2} \bar{N} \tau N \cdot \partial_{\mu}\left(\bar{N} S^{\mu} \tau N\right)-\frac{m_{\pi}^{2} \bar{g}_{0}}{\delta m_{N} F_{\pi}} \pi^{2} \pi_{3}+\ldots
\end{aligned}
$$

The first term defines the isoscalar $\left(\bar{d}_{0}\right)$ and isovector $\left(\bar{d}_{1}\right)$ short-range contributions to the nucleon EDM. The second term is the PVTV pion-nucleon non-derivative interactions consisting of isoscalar $\left(\bar{g}_{0}\right)$ and isovector $\left(\bar{g}_{1}\right)$ pieces. The operators with coefficients $C_{1,2}$ are PVTV two-nucleon contact interactions that account for heavier-meson exchange and other short-range effects [4]. These operators do not have proper chiral-transformation properties by themselves; their chiral partners are lumped in the "...". The “..." include also operators that contribute to EDMs beyond LO.

The constraint of proper chiral symmetry also enters through the orders of magnitudes of the LECs. Assuming NDA, we expect the six chiral EFT LECs to have the magnitudes shown in Table 1 in terms of the LECs from Eq. (1). Not all the LECs appear in EDMs at LO for all sources. For example the $\alpha_{e m}$ suppression means that for the qEDMs only the $\bar{d}_{0,1}$ are important. The three-pion coupling contributes at LO only for LRFQ. The link to PCTC isospin breaking fixes at LO $\bar{g}_{0} / \bar{\theta}=\left(1-\varepsilon^{2}\right) \delta m_{N} /(2 \varepsilon) \simeq$ $3 \mathrm{MeV}$ [3], using lattice QCD input [18]. For all sources a third, isotensor non-derivative pion-nucleon coupling is relatively small. This is in stark contrast with the traditional approach based on only the three non-derivative pion-nucleon couplings [14]. 
Table 1. Expected magnitudes of the six relevant chiral EFT LECs for the various PVTV sources of dimension up to six. For simplicity I take $\varepsilon=\mathcal{O}(1)$. (Adapted from Ref. [5].)

\begin{tabular}{c|ccccc} 
Source & $\bar{\theta}$ & qCEDM & qEDM & $\{$ gCEDM, CIFQ $\}$ & LRFQ \\
\hline$M_{Q C D} \bar{d}_{0,1} / e$ & $\mathcal{O}\left(\bar{\theta} \frac{m_{\pi}^{2}}{M_{Q C D}^{2}}\right)$ & $\mathcal{O}\left(\tilde{\delta}_{i} \frac{m_{\pi}^{2}}{M_{T}^{2}}\right)$ & $\mathcal{O}\left(\delta_{0} \frac{m_{\pi}^{2}}{M_{T}^{2}}\right)$ & $\mathcal{O}\left(\left\{w, \sigma_{i}\right\} \frac{M_{Q C D}^{2}}{M_{T}^{2}}\right)$ & $\mathcal{O}\left(\xi \frac{M_{Q C D}^{2}}{M_{T}^{2}}\right)$ \\
$\bar{d}_{1} / \bar{d}_{0}$ & $\mathcal{O}(1)$ & $\mathcal{O}(1)$ & $\mathcal{O}\left(\frac{\delta_{3}}{\delta_{0}}\right)$ & $\mathcal{O}(1)$ & $\mathcal{O}(1)$ \\
$\bar{g}_{0} / M_{Q C D}$ & $\mathcal{O}\left(\bar{\theta} \frac{m_{\pi}^{2}}{M_{Q C D}^{2}}\right)$ & $\mathcal{O}\left(\tilde{\delta}_{i} \frac{m_{\pi}^{2}}{M_{T}^{2}}\right)$ & $\mathcal{O}\left(\delta_{i} \frac{\alpha_{e m}}{4 \pi} \frac{m_{\pi}^{2}}{M_{T}^{2}}\right)$ & $\mathcal{O}\left(\left\{w, \sigma_{i}\right\} \frac{m_{\pi}^{2}}{M_{T}^{2}}\right)$ & $\mathcal{O}\left(\xi \frac{M_{Q C D}^{2}}{M_{T}^{2}}\right)$ \\
$\bar{g}_{1} / \bar{g}_{0}$ & $\mathcal{O}\left(\frac{m_{\pi}^{2}}{M_{Q C D}^{2}}\right)$ & $\mathcal{O}\left(\frac{\tilde{\delta}_{3}}{\tilde{\delta}_{i}}\right)$ & $\mathcal{O}(1)$ & $\mathcal{O}(1)$ & $\mathcal{O}(1)$ \\
$M_{Q C D} F_{\pi}^{2} \bar{C}_{1,2}$ & $\mathcal{O}\left(\bar{\theta} \frac{m_{\pi}^{2}}{M_{Q C D}^{2}}\right)$ & $\mathcal{O}\left(\tilde{\delta}_{i} \frac{m_{\pi}^{2}}{M_{T}^{2}}\right)$ & $\mathcal{O}\left(\delta_{i} \frac{\alpha_{e m}}{4 \pi} \frac{m_{\pi}^{2}}{M_{T}^{2}}\right)$ & $\mathcal{O}\left(\left\{w, \sigma_{i}\right\} \frac{M_{Q C D}^{2}}{M_{T}^{2}}\right)$ & $\mathcal{O}\left(\xi \frac{M_{Q C D}^{2}}{M_{T}^{2}}\right)$
\end{tabular}

Table 2. Dependence of the EDMs of the neutron, proton, deuteron, helion, and triton on the LECs in chiral EFT at LO. The PCTC isospin-breaking pion-nucleon coupling $\beta_{1}$ is not well known, $\beta_{1}=(0 \pm 9) \cdot 10^{-3}$ [19]. The contributions from the three-pion coupling through a three-nucleon force [5], $\Delta_{3 ; t, h}$, relevant only for LRFQ, have not yet been calculated (Adapted from Refs. [11, 14].)

\begin{tabular}{c|cccccc} 
LEC & $\bar{d}_{0}$ & $\bar{d}_{1}$ & $\left(\bar{g}_{0} / F_{\pi}\right) e \mathrm{fm}$ & $\left(\bar{g}_{1} / F_{\pi}\right) e \mathrm{fm}$ & $\left(F_{\pi}^{3} \bar{C}_{1}\right) e \mathrm{fm}$ & $\left(F_{\pi}^{3} \bar{C}_{2}\right) e \mathrm{fm}$ \\
\hline$d_{n}$ & 1 & -1 & - & - & - & - \\
$d_{p}$ & 1 & 1 & - & - & - & - \\
$d_{d}$ & 2 & 0 & $0.0002-0.07 \beta_{1}$ & -0.2 & - & - \\
$d_{h}$ & 0.83 & -0.93 & $-0.1+\Delta_{3 ; h}$ & -0.2 & -0.01 & 0.02 \\
$d_{t}$ & 0.85 & 0.95 & $0.1+\Delta_{3 ; t}$ & -0.2 & 0.01 & -0.02
\end{tabular}

\section{Electric dipole moments}

The Lagrangian 2 can be used together with the standard PCTC interactions $[6,7]$ for the calculation of EDMs in a controlled expansion in $Q / M_{Q C D}$, where $Q$ stands for the soft scales $m_{\pi}, F_{\pi}$, and, for nuclei, the binding momentum $\sqrt{m_{N} B}$. In Table 2 the dependence of the nucleon and light nuclear EDMs on the LECs from Eq. (2) are summarized. For a discussion of the uncertainties in the results, see Refs. [11, 14].

For a single nucleon, amplitudes are strictly perturbative. For all sources the short-range nucleon EDMs $\bar{d}_{0,1}$ contribute at LO, while for some sources one-loop diagrams stemming from $\bar{g}_{0,1}$ also appear at LO. These loop diagrams provide both short-range contributions and non-analytic pion-mass dependence. Assuming no cancellations with analytic terms encoded in the LECs, the non-analytic terms can, at a reasonable renormalization scale, provide a lower bound $[8,9]$ on the nucleon EDM, which leads to the $\bar{\theta}$ bound quoted above. All these terms appear together in nuclear calculations, so in Table 2 I lump them all with $\bar{d}_{0,1}$.

The computation of light nuclear EDMs is more complicated because we need to account for the non-perturbative dynamics that leads to nuclear bound states. PVTV form factors involve essentially a sandwich between PCTC wavefunctions of either PVTV currents or PCTC currents together with the PVTV potential [4]. Because light nuclei are characterized by binding momenta somewhat smaller than $F_{\pi}$, pions can be treated perturbatively, with the LO consisting of only momentum-independent contact interactions. Analytical expressions for the deuteron EDM can be found in Ref. [10]. Alternatively, one 
Table 3. Expected magnitudes of the neutron EDM (in units of $e / m_{N}$ ) and of the EDM ratios proton to neutron, deuteron to neutron, helion to neutron, and triton to helion, for the PVTV sources of dimension up to six. (Adapted from Refs. [11, 14].)

\begin{tabular}{c|ccccc} 
Source & $\bar{\theta}$ & qCEDM & qEDM & $\{$ gCEDM, CIFQ $\}$ & LRFQ \\
\hline$m_{N} d_{n} / e$ & $\mathcal{O}\left(\frac{m_{\pi}^{2}}{M_{Q C D}^{2}} \bar{\theta}\right)$ & $\mathcal{O}\left(\frac{m_{\pi}^{2}}{M_{T}^{2}} \tilde{\delta}_{i}\right)$ & $\mathcal{O}\left(\frac{m_{\pi}^{2}}{M_{T}^{2}} \delta_{i}\right)$ & $\mathcal{O}\left(\frac{M_{Q C D}^{2}}{M_{T}^{2}}\left\{w, \sigma_{i}\right\}\right)$ & $\mathcal{O}\left(\frac{M_{Q C D}^{2}}{M_{T}^{2}} \xi\right)$ \\
$d_{p} / d_{n}$ & $\mathcal{O}(1)$ & $\mathcal{O}(1)$ & $\mathcal{O}(1)$ & $\mathcal{O}(1)$ & $\mathcal{O}(1)$ \\
$d_{d} / d_{n}$ & $\mathcal{O}(1)$ & $\mathcal{O}\left(\frac{M_{Q C D}^{2}}{Q^{2}}\right)$ & $\mathcal{O}(1)$ & $\mathcal{O}(1)$ & $\mathcal{O}\left(\frac{M_{Q C D}^{2}}{Q^{2}}\right)$ \\
$d_{h} / d_{n}$ & $\mathcal{O}\left(\frac{M_{Q C D}^{2}}{Q^{2}}\right)$ & $\mathcal{O}\left(\frac{M_{Q C D}^{2}}{Q^{2}}\right)$ & $\mathcal{O}(1)$ & $\mathcal{O}(1)$ & $\mathcal{O}\left(\frac{M_{Q C D}^{2}}{Q^{2}}\right)$ \\
$d_{t} / d_{h}$ & $\mathcal{O}(1)$ & $\mathcal{O}(1)$ & $\mathcal{O}(1)$ & $\mathcal{O}(1)$ & $\mathcal{O}(1)$
\end{tabular}

can instead account for one-pion exchange non-perturbatively at the cost of a more complicated structure for the short-range interactions. Details are still being worked out at higher orders, so in Ref. [11] the EDMs of the deuteron, helion and triton have been estimated with high-accuracy phenomenological potentials instead. Results for the deuteron, which were confirmed for $\bar{\theta}$ [12], are in good agreement overall with perturbative-pion results. Another calculation for triton and helion [13] differed in a few matrix elements, a discrepancy that remains to be resolved. The estimates in Table 2 are averages of various calculations.

We can now combine the results of Tables 1 and 2 to obtain the sensitivity of each EDM to the underlying sources, see Table 3 . Their common invariance under chiral symmetry means that qCEDM and CIFQ cannot be separated without more information. Otherwise, the texture of this table underlines the argument [11] that light nuclei act as a "chiral filter" for the various PVTV sources. Of course, a measurement of the neutron EDM alone could be due to a $\bar{\theta}$ term of just the right magnitude, or to any of the dimension-six sources, although if all dimensionless factors were equal, gCEDM, CIFQ, and LRFQ would be favored because they require no chirality flip. Nuclear effects are most significant for $\bar{\theta}$, qCEDM, and LRFQ. Just on the basis of orders of magnitude, we see that a large tri-nucleon EDM compared to a nucleon EDM would point to them as possibly dominant sources, while a large $\left|d_{d}\right|$ compared to $\left|d_{n}\right|$ would be suggestive of just the qCEDM and LRFQ. Bounds on nuclear EDMs would provide tighter bounds on this physics than comparable bounds on nucleon EDMs.

We can infer more information about PVTV sources when we take into account that the relative importance of various pion-nucleon and short-range interactions is not the same for all sources [11]. For qCEDM and LRFQ, the expected dominance of nuclear effects comes from pion exchange due to both $\bar{g}_{0,1}$ couplings, while only $\bar{g}_{0}$ is present at LO for $\bar{\theta}$. The isoscalar coupling $\bar{g}_{0}$ approximately cancels in $d_{h}+d_{t}$, so while for the qCEDM $d_{h}+d_{t} \simeq 3 d_{d}$, for $\bar{\theta}$ we have instead $d_{h}+d_{t} \simeq 0.8\left(d_{n}+d_{p}\right)$. Effects of LRFQ can only be separated from qCEDM if its PVTV three-nucleon potential turns out to be significant. For the qEDM, where nuclear effects are much smaller, $d_{h}+d_{t} \simeq 0.8\left(d_{n}+d_{p}\right)$ also holds but in addition one expects $d_{h}-d_{t} \simeq 0.9\left(d_{n}-d_{p}\right)$. The situation is most complicated for gCEDM and CIFQ, for which nuclear effects are significant for both $A=2,3$, but they depend in the deuteron only on $\bar{g}_{1}$ while in the tri-nucleon $\bar{g}_{0}$ and $\bar{C}_{1,2}$ contribute as well. In this case $d_{h}+d_{t} \simeq 3 d_{d}-2\left(d_{n}+d_{p}\right)$. By confronting these relations, measurements of light nuclear EDMs, particularly if they include the triton, could shed light on the mechanism of $T$ violation [11]. 


\section{Conclusion}

EFTs provide a framework to describe the $T$-violating form factors of the nucleon and light nuclei systematically. (Using these techniques the PVTV magnetic [10,20] and PCTV toroidal [21] quadrupole form factors of the deuteron have been calculated as well, but they are unlikely to be measured.) Thanks to chiral symmetry, different $T$-violation sources produce different interactions at low energies. Experimental detection of the EDMs should allow for the identification of the dominant source, if one exists.

I am grateful to the organizers for the invitation to a forward-looking conference, and to my collaborators for their insight into the research reported here. This work was supported in part by the US DOE under grant DE-FG0204ER41338 and by the Université Paris Sud through a grant Attractivité 2013.

\section{References}

[1] C.A. Baker et al., Phys. Rev. Lett. 97, 131801 (2006)

[2] J. Pretz, Hyperfine Interact. 214, 111 (2013)

[3] E. Mereghetti, W. Hockings, and U. van Kolck, Annals Phys. 325, 2363 (2010)

[4] C. Maekawa, E. Mereghetti, J. de Vries, and U. van Kolck, Nucl. Phys. A 872, 117 (2011)

[5] J. de Vries, E. Mereghetti, R.G.E. Timmermans, and U. van Kolck, Annals Phys. 338, 50 (2013)

[6] P.F. Bedaque and U. van Kolck, Ann. Rev. Nucl. Part. Sci. 52, 339 (2002)

[7] V. Bernard, N. Kaiser, and U.-G Meißner, Int. J. Mod. Phys. E 4, 193 (1995)

[8] R.J. Crewther, P. Di Vecchia, G. Veneziano, and E. Witten, Phys. Lett. B 88, 123 (1979); 91, 487(E) (1980); W.H. Hockings and U. van Kolck, Phys. Lett. B 605, 273 (2005); S. Narison, Phys. Lett. B 666, 455 (2008); K. Ottnad, B. Kubis, U.-G. Meißner, and F.-K. Guo, Phys. Lett. B 687, 42 (2010)

[9] J. de Vries, E. Mereghetti, R.G.E. Timmermans, and U. van Kolck, Phys. Lett. B 695, 268 (2011); E. Mereghetti, J. de Vries, W.H. Hockings, C.M. Maekawa, and U. van Kolck, Phys. Lett. B 696, $97(2011)$

[10] J. de Vries, E. Mereghetti, R.G.E. Timmermans, and U. van Kolck, Phys. Rev. Lett. 107, 091804 (2011)

[11] J. de Vries, R. Higa, C.-P. Liu, E. Mereghetti, I. Stetcu, R.G.E. Timmermans, and U. van Kolck, Phys. Rev. C 84, 065501 (2011)

[12] J. Bsaisou, C. Hanhart, S. Liebig, U.-G. Meißner, A. Nogga, and A. Wirzba, Eur. Phys. J. A 49, $31(2013)$

[13] Y.-H. Song, R. Lazauskas, and V. Gudkov, Phys. Rev. C 87, 015501 (2013)

[14] J. Engel, M.J. Ramsey-Musolf, and U. van Kolck, Prog. Part. Nucl. Phys. 71, 21 (2013)

[15] B. Grzadkowski, M. Iskrzynski, M. Misiak, and J. Rosiek, JHEP 1010, 085 (2010)

[16] V. Baluni, Phys. Rev. D 19, 2227 (1979)

[17] W. Dekens and J. de Vries, JHEP 1305, 149 (2013)

[18] S.R. Beane, K. Orginos, and M.J. Savage, Nucl. Phys. B 768, 38 (2007)

[19] U. van Kolck, J.L. Friar, and J.T. Goldman, Phys. Lett. B 371, 169 (1996); U. van Kolck, M.C.M. Rentmeester, J.L. Friar, J.T. Goldman, and J.J. de Swart, Phys. Rev. Lett. 80, 4386 (1998)

[20] C.-P. Liu, J. de Vries, E. Mereghetti, R.G.E. Timmermans, and U. van Kolck, Phys. Lett. B 713, 447 (2012)

[21] E. Mereghetti, J. de Vries, R.G.E. Timmermans, and U. van Kolck, Phys. Rev. C 88, 034001 (2013) 